\title{
CLINICAL AND EXPERIMENTAL STUDY OF GAALAS PHOTOTHERAPY FOR TEMPOROMANDIBULAR DISORDERS
}

\author{
S. Nencheva-Svechtarova ${ }^{1}$, V. Svechtarov ${ }^{2}$, A. Gisbrecht ${ }^{3}$ and Tz. Uzunov ${ }^{4}$ \\ ${ }^{1}$ Center for Integrated Dental Medicine, Faculty of Dental Medicine, Medical University - Sofia \\ ${ }^{2}$ Department of Oral and Maxillofacial Surgery, Faculty of Dental Medicine, Medical University - Sofia \\ ${ }^{3}$ Institute of Electronics, Bulgarian Academy of Sciences \\ ${ }^{4}$ Department of Conservative Dentistry, Faculty of Dental Medicine, Medical University - Sofia
}

Summary. The objective of this study was to test the clinical effectiveness of the gallium-aluminum-arsenide laser (GaAIAs; $785 \mathrm{~nm}$ ) and superluminiscent diodes $(633 \mathrm{~nm})$ phototherapy (MedX 1100 device) for the treatment of patients with temporomandibular disorders and myofascial pain syndrome. The results demonstrated a positive effect in pain relief. A significant reduction $(p<0.05)$ in the level of pain was observed for the temporomandibular joint and for the masseter muscles using paired samples t-test and Wilcoxon signed rank test. The experimental study on pork muscle samples showed that a) the main part of laser radiation is absorbed by the tissue in thin layer of 3-4 mm, b) in the spectral region $650-950 \mathrm{~nm}$ the intensity of light penetration is about 0.2-0.25 percent of the initial light intensity.

Key words: gallium-aluminum-arsenide laser, temporomandibular disorders, laser penetration depth

\section{INTRODUCTION}

A wide variety of light sources such as lasers, superluminiscent diodes (SLD) or light-emitting diodes (LED) may be used for therapeutic pur poses. The energy emitted provides temporary increase in microcirculation, temporary relief of minor muscle and joint aches, pain and stiffness, relaxation of muscles, muscle spasms, minor pain and stiffness associated with arthritis. The most commonly used low-level light sources operate in the $780-870 \mathrm{~nm}$ spectral range, where light penetration depth is considered to be up to $5 \mathrm{~cm}$ due to a lower degree of absorption. The key to an effective treatment is the adequacy of the opti- 
cal parameters of the light and its dosage delivered to the tissue. Among the large amount of scientific data in the literature, there is practically limited information on the clinical outcome of low level laser therapy (LLLT) of patients with chronic temporomandibular and myofascial complaints. Certain works report only fragmentary and incomplete data.

Maia, M. L. et al. [1] summarizes fourteen studies on the use of low level laser therapy for treatment of temporomandibular disorders (TMD) from which thirteen show a reduction in pain levels. In nine of them the pain reduction is registered only in the experimental group, while for the remaining four the effect is observed for both the experimental and the placebo groups. However, these studies use various parameters and calibration techniques, thus further analysis is needed for conclusions. Venezian et al. [2] evaluate the effect of diode lasers on pain to palpation and electromyographic (EMG) activity of the masseter and anterior temporalis muscles. The authors show that only the active doses cause statistically significant reduction in pain level in all regions of the explored muscles. Carrasco et al. [3] applied GaAIAs $(780 \mathrm{~nm})$ laser to patients with myofascial pain syndrome and found a significant pain reduction. Fikachkova et al. [4] treated 61 patients with temporomandibular dysfunction and myofascial pain with 10 doses of $10 \mathrm{~J} / \mathrm{cm}^{2}$ and $15 \mathrm{~J} / \mathrm{cm}^{2}$ using GaAlAs laser with power output of $400 \mathrm{~mW}$ at a wavelength of $830 \mathrm{~nm}$. Laser therapy was applied in the areas of painful myofascial trigger points. The results showed that the laser therapy is very effective in the treatment of chronic TMD. According to Chang et al.'s [6] meta-analysis on 7 articles of the clinical effects of LLLT on TMD pain, the analgesic effect achieved with wavelengths of $830 \mathrm{~nm}$ and $780 \mathrm{~nm}$ is moderate or superior. Other studies have also reported similar results [8-12]. Emshoff et al. [6] argued that laser treatment with wavelength of $632.8 \mathrm{~nm}$ did not generate the desired analgesic effects.

As shown above, publications regarding the application of infrared and red light for the treatment of patients with temporomandibular disorders and myofascial pain syndrome are not sufficient. As it is well known, the analgesic effect of LLLT strongly depends on the laser radiation dosage. That is why at low dose rate there may be no effect. Additional clinical research regarding the treatment effect, wavelength and application methods is needed.

\section{AIM OF THE STUDY}

The aim of this study is to evaluate the effectiveness of GaAlAs laser (785 nm) and superluminiscent diode red light $(633 \mathrm{~nm})$ on pain intensity reduction in individuals with chronic temporomandibular and myofascial disorders. Our next aim was to find a biological reason for the significant reduction of pain in some organs but not in others.

\section{MATERIAL AND METHODS}

The present study included 45 patients, aged between 17 and 70 years, examined during the period 2010-2013. All patients had clinical symptoms of chronic joint and/or myofascial pain caused by different etiological factors. Chronic myofas- 
cial pain is defined as a painful condition existing for at least three months. Data is collected based on the presence of pain on palpation in the TM joint, masticatory muscles, sternocleidomastoids, trapezius and the regional orofacial structures. The diagnosis and classification of the disorders is done using Standardized Axis 1 of RDC/TMD (Research Diagnosis Criteria for Tempomandibular Disorders, 2010), which includes questionnaire about the history of the disease and methods for clinical examination.

Flat and volumetric palpation of the masticatory and cervical muscles is used for localization of the myogenic trigger points as well as palpation of the joints and isometric contraction tests for the lateral and medial pterygoids. The data for pain localization and its intensity was recorded for each individual. Outcome measurements included VAS pain scores. The data file analysed includes pain intensity of the temporomandibular joint - uni- and bilaterally, the temporal muscles, masseters, medial pterygoids, posterior digastrics, lateral pterygoids, sternocleidomastoideus and trapezius.

The pain intensity measurements were taken before and after the completion of six sessions ( 3 times per week) of photo therapy. Trigger point oriented laser applications (MedX 1100 console with three GaAlAs laser diodes, $785 \mathrm{~nm}, 3$ × $33 \mathrm{~mW}$, $100 \mathrm{~s}, 8 \mathrm{~J} / \mathrm{cm}^{2}$ per spot) were performed for the temporomandibular joints, the temporal, lateral, medial pterygoids and sternocleidomastoid muscles; a combination of laser and SLD red light $\left(633 \mathrm{~nm}, 200 \mathrm{~mW}, 300 \mathrm{~s}, 8 \mathrm{~J} / \mathrm{cm}^{2}\right.$ per spot) was applied for masseter and trapezius muscles. Both paired t-test and the corresponding nonparametric Wilcoxon signed rank test are used. The difference in pain (before and after therapy) is the variable of interest. The null hypothesis is that the difference is equal to zero. SPSS statistical software was utilized. For the paired t-test we used the paired samples for each patient before and after the laser therapy.

To determine how deep laser light penetrate in the tissue we carried out some model experiments. The study was performed on random samples of pork muscles with different thickness. Quantitative determination of the relative laser light penetration in the tissue was carried out using a precise microspectrometer S2000 ("Ocean Optics Inc"). It measured the intensity of the light passing through the sample with $6 \mathrm{~mm}$ thickness. The spectra were analyzed and graphically displayed by means of computer programs Origin 75 and Corel.

\section{RESULTS}

The percent of women in the study is $84 \%$; the mean age is 42,16 (SD 14 , 2 ). The results from the paired t-test and the nonparametric test are summarized in Table 1.

Experimental results: The laser beam is pointed perpendicularly through the pattern to the formed flat region of the sample. On the opposite side the transmitted light is collected into the optical fiber. Fig. 1 shows the photography visualization of light propagation via tissue sample with $6 \mathrm{~mm}$ thickness. 
Table 1. Paired t-test results and Wilxocon signed rank test

\begin{tabular}{|l|c|c|c|}
\hline Organ & $\mathrm{t}$-statistic & $\mathrm{p}$-value & Wilxocon test \\
\hline Temporomandibular joint, unilateral & 3.110 & 0.005 & reject H0 \\
\hline Temporomandibular joint, bilateral & 5.707 & 0.001 & reject H0 \\
\hline Masseter, unilateral & 3.211 & 0.03 & reject H0 \\
\hline Masseter, bilateral & 3.067 & 0.005 & reject H0 \\
\hline Sternocleidomastoideus, unilateral & 1.788 & 0.84 & retain H0 \\
\hline $\begin{array}{l}\text { Temporalis, Medial pterygoids, Posterior digastrics, } \\
\text { Lateral pterygoids, Trapezius, uni- and bilateral }\end{array}$ & 1.000 & 0.326 & retain H0 \\
\hline
\end{tabular}

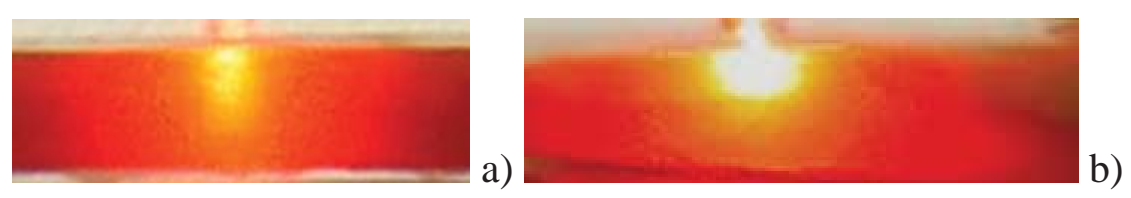

Fig. 1. Visualization of light propagation via tissue

The qualitative analysis shows that the scattering of light varies in the different tissues. It is more directly spread in muscle (Fig. 1a) and more diffuse in fat tissue (Fig. 1b), but in general, the main part of laser radiation is absorbed by the tissue in thin layer of 3-4 $\mathrm{mm}$.

The data also shows that the penetration of light depends on the wavelength; the higher penetration is observed in the red/near-infrared region of the spectrum and the relatively low - in the blue region. Particularly, in the spectral region 650-950 nm the intensity of light penetration is about 0.2-0.25 percent of the initial light intensity, whereas in the blue and green spectral region it is about 0.05-0.1 percent (Fig. 2).

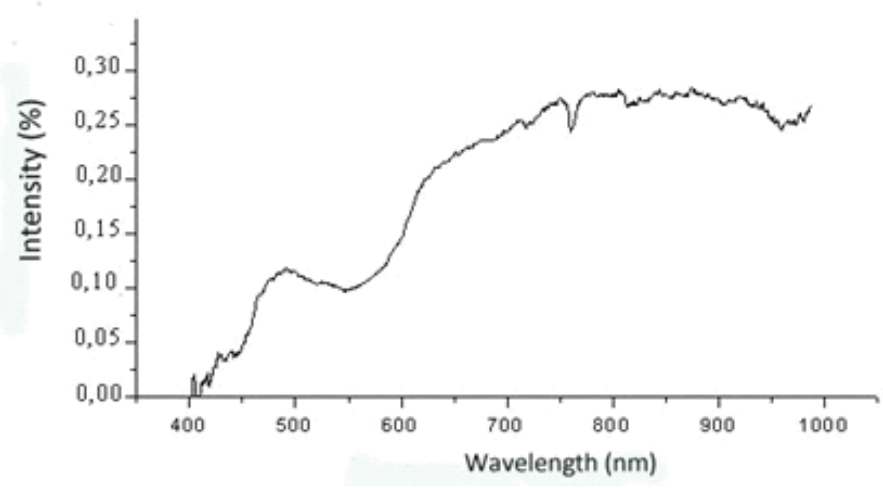

Fig. 2. The spectral distribution of the transmitted laser light 


\section{DISCUSSION}

This study shows that GaAlAs phototherapy provides statistically significant reduction of pain symptoms in the temporomandibular joints and in the masseters. The results of the paired t-test are similar with the results from the nonparametric test. It should be emphasized that combined laser and SLD phototherapy is very effective particularly in the reduction of masseter myofascial pain, which is a masticatory muscle strongly affected by TMJ dysfunction with various etiology. However, there are areas for which we do not observe statistical significance, e.g. temporalis, medial and lateral pterygoid muscles, sternocleidomastoideus and trapezius.

Concerning the light penetration depth we would like to point out that these measurements are applicable to homogeneous samples, like our model experiment. Real morphology of living tissue is more complicated. For example, Uzunov et al. [7] were investigated the penetration of the radiation of different LEDs in the visible spectral range into oral tissues. It is shown that in heterogeneous structures due to multiple reflections of laser light from the edge of different tissues can result in significant reduction of light penetration. Therefore, the success of the phototherapy is strongly related to the optical properties and the depth of tissues. That is why in some cases our treatment had no effect.

\section{CONCLUSIONS}

Phototherapy with laser light in the red/near-infrared spectral region demonstrated positive results in the relief of pain. The analgesia of LLLT depends on the laser's wavelength and the radiation dosage when using LLLT to treat TMJ pain. The key to an effective treatment is the adequacy of the dosage delivered to the tissue. Because of the higher light penetration in the red/near-infrared region it is recommended for use in phototherapy treatment of painful symptoms. In order to choose the proper dosage of radiation in phototherapy, a knowledge for the penetration properties of the tissue configuration is required.

The obtained experimental results can be used in dental practice for determination of the treatment efficiency of the therapy and the specific limits for each tissue-laser combination. A goal for future research is to find a suitable method for optical delivery of laser light to the target organs, e.g. via intraoral approach, especially for the deeply located masticatory muscles like lateral and medial pterygoids.

\section{REFERENCES}

1. M a ia, M. L. et al. Effect of low-level laser therapy on pain levels in patients with temporomandibular disorders: a systematic review. - J. Appl. Oral. Sci., 20, 2012, № 6, 594-602.

2. Venezian, G. C.et al. Low level effects on pain to palpation and electromyoigraphic activity in TMD patients: a double-blind, randomized, placebo-controlled study. - Granio, 28, 2010, № 2, 84-91.

3. Carrasco, T. G. et al. Evaluation of low intensity laser therapy in myofascial pain syndrome. Cranio, 27, 2009, № 4, 243-247. 
4. Fikachkova, H. et al. Arthralgia of the temporomandibular joint and low-level laser therapy Photomed Laser Surg., 24, 2006, № 4, 522-527.

5. Chang, W. D. et al. A Meta-analysis of Clinical Effects of Low-level Laser Therapy on Temporomandibular Joint Pain. - J. Phys. Ther. Sci., 26, 2014, № 8, 1297-1300.

6. E m sh off, R. et al. Low-level laser therapy for treatment of temporomandibular joint pain: a double-blind and placebo-controlled trial. - Oral Surg. Oral Med. Oral Pathol. Oral Radiol. Endod., 105, 2008, № 4, 452-456.

7. Uzunov, Tz. et al. Comparative investigation of the penetration of different wavelength visible LED radiation into dental tissue"- Proc. SPIE 7027, 15th International School on Quantum Electronics: Laser Physics and Applications, 70271C.

8. Carra s c o, T. G.et al. Low intensity laser therapy in temporomandibular disorder: a phase II double-blind study. - Cranio, 26, 2008, № 4, 274-281.

9. Fiká cková, H. et al. Effectiveness of low-level laser therapy in temporomandibular joint disorders: a placebo-controlled study. - Photomed. Laser Surg., 25, 2007, № 4, 297-303.

10. Mazzet to, M. O.et al. Low intensity laser application in temporomandibular disorders: a phase I double-blind study. - Cranio, 25, 2007, № 3, 186-192.

11. Cetine r, S., S. A. Kahraman et S. Yücetaş. Evaluation of low-level laser therapy in the treatment of temporomandibular disorders. - Photomed. Laser Surg., 24, 2006, № 5, 637-641.

12. Venancio, R. A., C. M. Camparis et R. F. Lizarelli. Low intensity laser therapy in the treatment of temporomandibular disorders: a double-blind study. - J. Oral Rehabil., 32, 2005, № 11, 800-807.

\section{Corresponding author:}

Dr. Savina Nencheva-Svechtarova

Faculty of Dental Medicine

1, Sv. Georgi Sofiiski St

BG - 1431 Sofia

Tel. + 359886736798

e-mail: savinanencheva@gmail.com 